\title{
EFEITO DA MASSOTERAPIA NOS SINTOMAS DA DOR MUSCULAR TARDIA
}

\section{Effects of massage on delayed-onset muscle soreness}

\author{
Juliane Cabral Silva ${ }^{1}$, Charles de Souza Vieira ${ }^{1}$, Lucas Pereira Lopes de Souza ${ }^{1}$, Rodrigo Cappato de Araújo ${ }^{1}$ \\ ${ }^{1}$ Universidade de Pernambuco, Petrolina/PE
}

Resumo: A dor muscular tardia está associada a exercícios não-acostumados principalmente de natureza excêntrica, tendo seu pico entre 24-72h e caracteriza-se pelo aparecimento de dor, rigidez, edema e redução na amplitude de movimento (ADM). O estudo tem como objetivo observar os efeitos da massoterapia sobre os sintomas da dor muscular tardia. Vinte voluntários foram divididos de forma aleatória em dois grupos contendo dez pessoas cada, onde ambos realizaram 3 séries com 10 contrações excêntricas sub-máximas do músculo tríceps sural, sendo um grupo controle e outro submetido a um protocolo de massoterapia logo após o exercício. Foram avaliadas ADM de joelho e tornozelo, perimetria da perna e a dor através da escala visual analógica. Essas avaliações ocorreram antes, após, 24, 48 e 72 horas após o protocolo de exercício. A análise estatística foi feita por meio do teste ANOVA pos hoc Tukey, com nível de significância de $5 \%$. O grupo controle e o grupo de massoterapia apresentaram pico de dor 48 horas após o exercício. $\mathrm{O}$ grupo controle ainda apresentou diminuição do movimento de extensão ativa da articulação do joelho, enquanto o grupo que sofreu a intervenção não apresentou alterações significantes na amplitude de movimento. Conclui-se que a técnica é eficaz para evitar a redução de amplitude de extensão ativa do joelho, porém não reduz a dor após um exercício excêntrico não acostumado.

Palavras-chave: Dor muscular tardia, massoterapia, exercício excêntrico.

\begin{abstract}
Delayed on-set muscle soreness is associated with unaccustomed exercise mainly eccentric nature, the intensity of soreness increases during the first 24-72 hours and it is characterized by appearance of pain, stiffness, swelling and reduction in range of motion (ROM). The objective of this study was to observe the effect of the massage on the symptoms of delayed on-set muscle soreness. Twenty volunteers were randomly assigned to either a massage or control group. The volunteers realized 3 sets of 10 submaximal eccentric contractions of the triceps sural muscle. ROM of knee and ankle, perimetry of the leg and pain were evaluated. These evaluations occurred before, after, 24,48 and 72 hours after the exercise protocol. The statistics analysis was evaluated by ANOVA and Tukey pos hoc test. Statistical significance was set at $p<0.05$. The control and massage groups presented peak of pain 48 hours after the exercise. The group control still presented reduction of the movement of active extension of the knee joint, while the group that suffered the intervention did not present significant alterations in the range of motion. The massage technique is efficient to prevent the reduction of range of active knee extension, however does not reduce pain after an unaccustomed eccentric exercise.
\end{abstract}

Key-words: Delayed muscle soreness, massage, eccentric exercise.

Aceito em 22/10/2009 - Rev. Educ. Fís. 2010 - 20-26. Rio de Janeiro - RJ - Brasil

\section{INTRODUÇÃO}

Dor muscular tardia (DMT) é um desconforto no músculo esquelético após exercícios excêntricos ${ }^{(1,2,3,4)}$ ou isométrico intenso ${ }^{(2)}$. É classificada como um tipo de lesão por tensão muscular ${ }^{(5)}$, apresentando redução da amplitude de movimento articular ${ }^{(6,7,8)}$, edema ${ }^{(9)}$ e sensibilidade ou rigidez a palpação e/ou movimento $^{(5)}$. A intensidade do desconforto aumenta nas primeiras 24 horas após o término do exercício, apresentando um pico nas 48 horas, diminuindo e eventualmente desaparecendo entre 5-7 dias pós-exercício ${ }^{(10}$, 11,12).
00 fenômeno da dor muscular tardia é explicado como sendo uma tensão mecânica gerada no tecido muscular e no tecido conectivo durante as contrações excêntricas por uma diminuição na atividade das unidades motoras causando microlesões que desencadeiam um influxo de íons cálcio do interstício para o interior da fibra muscular, bem como a marginação de neutrófilos. O excesso desse íon antecipa uma fase autogênica, resultando uma lesão progressiva das miofibrilas e da membrana plasmática, gerando uma reação inflamatória local ${ }^{(13)}$, conseqüentemente 0 aparecimento do edema, que irá comprimir as terminações nervosas gerando dor ${ }^{(14)}$, 
reduzindo a amplitude de movimento e a capacidade de gerar força(15).

Com o surgimento destes sintomas, haverá uma alteração biomecânica nos músculos e articulações afetados, limitando a funcionalidade dessas estruturas e conseqüentemente a execução das atividades de vida diária em indivíduos que praticam exercícios físicos por lazer, bem como, pode acarretar impacto negativo no desempenho de atletas submetidos às intensas temporadas de treinamentos e competições. Por isso, são importantes estudos que busquem minimizar os sintomas relacionados a este quadro, a fim de melhorar a qualidade de vida da população e o desempenho dos atletas ${ }^{(15)}$.

Várias estratégias de tratamento para o alivio dos sintomas da DMT tem sido testadas por diferentes pesquisadores, sempre com o objetivo de restaurar a função máxima do músculo o mais precoce possível e reduzir a magnitude da lesão inicial. Para isso, os estudos tem utilizado a crioterapia ${ }^{(16)}$, o exercício de alongamento muscular antes e/ ou pós-exercicio(5), administração de drogas anti-inflamatórias $^{(17)}$, aplicação do ultra-som ${ }^{(18)}$ e outras correntes elétricas, assim como a massoterapia(8). A massagem muscular pode ser uma terapia alternativa, já que é popular e possivelmente efetiva poraumentara quantidade de fluxo sanguíneo oxigenado e linfático para a área lesionada, diminui produção de edemas e reduz tônus muscular ${ }^{(19)}$. Estudos referem diminuição da DMT quando a massagem é aplicada 2 horas após exercício ${ }^{(20,21,22)}$, porém não há referência quanto a evitar redução de amplitude de movimento, nem a aplicação da técnica apenas imediatamente após o exercício excêntrico. Quanto às técnicas mais utilizadas temos a Petrissage e Effleurage, variando no tempo de aplicação de cada uma.

Então associando estas técnicas e o alisamento superficial durante 30 minutos, este estudo diferencia-se dos demais quanto ao início da aplicação da massagem, bem como no tempo de cada técnica utilizada; e objetiva analisar os efeitos da massagem no edema, na redução da amplitude de movimento e dor, os quais são sinais e sintomas presentes após a realização de tarefas excêntricas intensas.

\section{METODOLOGIA}

\section{Sujeitos}

Trata-se de um estudo clínico randomizado controlado, que foi realizado em 20 voluntários de ambos os sexos, com idade variando entre $18 \mathrm{e}$ 30 anos, todos sedentários e que foram divididos em dois grupos: Grupo Controle composto por 10 indivíduos, os quais não sofreram intervenção, mas participaram de todas as etapas de avaliação; e Grupo Massoterapia composto por 10 indivíduos, estes foram submetidos a um protocolo de massoterapia. Foram incluídos os voluntários que não estivessem participando de nenhum programa de exercício ou qualquer outra atividade física há pelo menos três meses e excluídos os que apresentaram história de lesões musculoesqueléticas e/ou cirurgias nos membros inferiores. Todos os voluntários receberam informações para participar do estudo, leram e assinaram um termo de consentimento, segundo a resolução $n^{\circ}$ 196/96 do Conselho Nacional da Saúde. O estudo foi aprovado pelo comitê de ética e pesquisa da Universidade de Pernambuco.

\section{Procedimentos}

Inicialmente, cada voluntário foi submetido a uma avaliação física, na qual foram colhidos dados pessoais e antropométricos, abordando questões relacionadas à história de traumas prévios nos membros inferiores, além de um questionamento sobre as atividades físicas realizadas pelos voluntários. Após isso, mensurou-se a dor através do método EVA, escala visual analógica, que é composta por uma reta medindo 10 centímetros, na qual o indivíduo marca um ponto de acordo com a intensidade de sua dor, sendo zero, ausência de dor e dez a máxima dor referida.

A amplitude de movimento passiva e ativa das articulações do joelho e tornozelo foi avaliada por meio de um goniômetro universal de $360^{\circ}$ da marca CARCI. No movimento de flexão do joelho o voluntário encontrava-se inicialmente em decúbito ventral com os joelhos estendidos, com o goniômetro posicionado no eixo da articulação do joelho, o braço fixo posicionado em paralelo ao fêmur e o braço móvel acompanhava movimento de flexão da perna. A extensão do joelho foi avaliada com o voluntário em decúbito 
ventral com o membro inferior estendido, com goniômetro posicionado no eixo da articulação do joelho, o braço fixo e móvel foram colocados em paralelo à coxa e à perna, respectivamente $e$ solicitado que o voluntário realizasse a extensão máxima do joelho. Para a mensuração dos movimentos de dorsi-flexão e na flexão-plantar do tornozelo, cada voluntário foi avaliado em decúbito ventral com o joelho fletido a $90^{\circ}$ e o tornozelo em neutro (posição anatômica), com goniômetro posicionado no eixo da articulação, o braço fixo paralelo à fíbula e o braço móvel na direção do quinto metatarso. Todas as medições foram realizadas por um único avaliador $e$ repetidas três vezes para que se obtivesse um valor médio.

Para a avaliação da presença de edema na região da perna, foi utilizada a técnica de perimetria, utilizando uma fita métrica para a mensuração do diâmetro de seis pontos com cinco centímetros de distância entre eles, partindo da tuberosidade da tíbia até o maléolo lateral. A avaliação da dor, amplitude de movimento e edema foi feita antes, logo após e $24 \mathrm{~h}, 48 \mathrm{~h}$ e $72 \mathrm{~h}$ após o exercício.

Após a avaliação o voluntário foi submetido a um protocolo de indução de dor para a musculatura do tríceps sural direito. Para a prática desse protocolo, foi calculada a resistência máxima do indivíduo através do teste de RM onde, $1 \mathrm{RM}$ é definido como a maior carga que pode ser movida por uma amplitude específica de movimento uma única vez e com execução correta; após ter sido definido a $1 \mathrm{RM}$, foram calculados $90 \%$ da mesma, definindo assim a carga máxima a ser utilizada no exercício.

Os voluntários realizaram o exercício no aparelho da marca Nakagym, modelo Smith Machine 1.18 NK6020, ficando em posição ortostática com os pés apoiadas sobre uma plataforma declinada a 15 graus e com a carga apoiada nos ombros. Inicialmente, foi realizado com apoio bipodal a contração concêntrica do músculo tríceps sural (FIGURA $1 A$ ), posteriormente, foi realizada com apoio unipodal direito, a contração excêntrica (FIGURA 1B). Foram realizadas três seções de dez repetições desse exercício a fim de provocar as microlesões necessárias para o aparecimento da dor muscular tardia.
FIGURA 1. FASE CONCÊNTRICA DO MOVIMENTO REALIZADA COM APOIO BIPODAL (A) E FASE EXCÊNTRICA REALIZADA COM APOIO UNIPODAL (B).

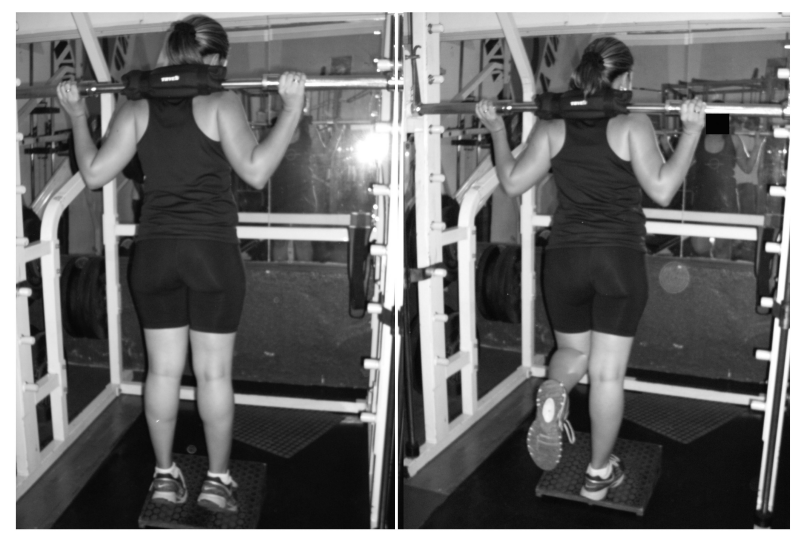

Nos voluntários do Grupo Massoterapia foram realizados 30 minutos de massagem, sendo aplicada, 5 minutos da técnica de alisamento superficial, realizada de forma lenta e suave e em qualquer direção; em seguida foram aplicados 15 minutos de manipulações de Pétrissage, nessa técnica estão incluídos os movimentos de amassamento, torcedura e rolamento da pele. $O$ amassamento com os polegares, aplicado durante 5 minutos, foi realizado em um movimento circular e de forma lenta, sendo a pressão aplicada durante metade do movimento e liberada durante a outra metade, para que ocorresse o relaxamento da pressão. $\mathrm{Na}$ torcedura, os polegares assumiram uma posição de grande abdução em relação aos dedos, os tecidos foram agarrados com ambas as mãos, levantados e em seguida comprimidos entre os dedos e os polegares da mão oposta; as mãos operaram transversalmente às fibras musculares, aplicada com uma velocidade de lenta a média e por 5 minutos. $O$ rolamento foi feito de forma lenta, durante 5 minutos, e consistiu em produzir uma prega de pele entre os dedos e os polegares, os polegares comprimiram os tecidos na direção dos dedos, rolando-os em um movimento ondular, e afastando-os do terapeuta. Para encerrar foram aplicados 10 minutos da técnica effleurage, administrada na direção do fluxo venoso e linfático com movimentos suaves e rítmicos direcionando para os linfonodos poplíteos e que consiste em manobras de deslizamento superficial realizada com a região palmar e dedos.

Todos os procedimentos de avaliação, bem como a realização de todas as técnicas 
de massagem foram realizados pelo mesmo fisioterapeuta, em todos os voluntários da pesquisa.

\section{Análise Estatística}

Todos os procedimentos estatísticos foram realizados por meio do programa SPSS versão 11.0. Antes da análise de cada variável, a normalidade na distribuição dos dados foi verificada por meio do teste de KolmogorovSmirnov. As comparações dos dados da EVA e perimetria foram realizadas por meio de uma análise de variância (ANOVA) e pos hoc Tukey, enquanto que os dados de amplitude de movimento foram analisados com o ANOVA de Friedman e pos hoc Dunn. Em todas as situações foi utilizado um nível de significância de $p \leq 0,05$.

\section{RESULTADOS}

O GRÁFICO 1 apresenta os escores da escala visual analógica (EVA) para os dois grupos avaliados. A comparação intragrupo demonstrou um aumento significativo $(p<0,01)$ dos níveis de dor 48 horas após a realização do exercício em ambos os grupos. No entanto, a análise intergrupo não apresentou diferença estatística, sugerindo ineficácia das técnicas utilizadas na prevenção da dor muscular tardia nesse tipo de exercício e no grupo muscular analisado.

GRÁFICO 1. COMPORTAMENTO DOS VALORES DA ESCALA VISUAL ANALÓGICA (EVA) AO LONGO DAS PRIMEIRAS 72 HORAS APÓS A REALIZAÇÃO DO PROTOCOLO DE EXERCÍCIO.

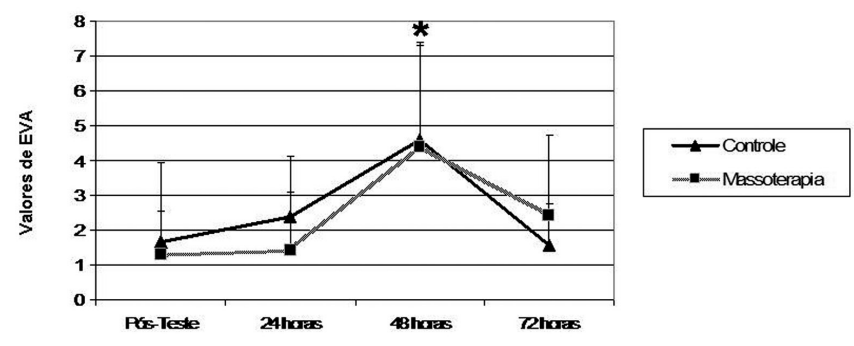

As TABELAS 1 e 2 apresentam os resultados absolutos, identificando os valores de amplitude articular de movimento e perimetria, respectivamente. Em relação à análise da perimetria, não foi possível observar a presença de edema em nenhum dos dois grupos ao longo do estudo. Já os valores de goniometria demonstraram uma diminuição da amplitude movimento de extensão do joelho no grupo controle, 48 horas após o exercício $(p=0,029)$, quando comparado aos valores pré-exercício. No entanto, o grupo submetido à técnica de massoterapia não apresentou diminuição da amplitude de movimento das articulações do joelho e tornozelo.

TABELA 1. MÉDIA E DESVIO-PADRÃO DOS VALORES DE AMPLITUDE DE MOVIMENTO DAS ARTICULAÇÕES DE JOELHO E TORNOZELO.

\begin{tabular}{|c|c|c|c|c|c|c|}
\hline & & $\begin{array}{c}\text { Pré- } \\
\text { exercício }\end{array}$ & $\begin{array}{c}\text { Pós- } \\
\text { exercício }\end{array}$ & 24 horas & 48 horas & 72 horas \\
\hline \multirow{4}{*}{$\begin{array}{c}\text { Grupo } \\
\text { Controle }\end{array}$} & $\begin{array}{l}\text { Flexão } \\
\text { joelho }\end{array}$ & $130,7 \pm 7,1$ & $131,2 \pm 5,2$ & $132,9 \pm 5,8$ & $133,5 \pm 9,7$ & $133,4 \pm 7,7$ \\
\hline & $\begin{array}{c}\text { Extensão } \\
\text { joelho }\end{array}$ & $180,4 \pm 1,2$ & $180,4 \pm 1,2$ & $180,3 \pm 1,3$ & $178,7 \pm 1,6^{*}$ & $179,6 \pm 0,8$ \\
\hline & $\begin{array}{l}\text { Flexão } \\
\text { Plantar }\end{array}$ & $57,8 \pm 15,6$ & $61,2 \pm 16,6$ & $61,1 \pm 14,9$ & $62,2 \pm 12,4$ & $62,6 \pm 15,4$ \\
\hline & $\begin{array}{l}\text { Dorsi } \\
\text { Flexão }\end{array}$ & $14,1 \pm 5,4$ & $15,2 \pm 5,1$ & $15,1 \pm 4,3$ & $16,6 \pm 6,2$ & $16,6 \pm 5,0$ \\
\hline \multirow{4}{*}{$\begin{array}{c}\text { Grupo } \\
\text { Massoterapia }\end{array}$} & $\begin{array}{l}\text { Flexão } \\
\text { joelho }\end{array}$ & $132,7 \pm 6,0$ & $130,3 \pm 6,0$ & $133,4 \pm 6,8$ & $129,9 \pm 5,9$ & $130,5 \pm 8,7$ \\
\hline & $\begin{array}{c}\text { Extensão } \\
\text { joelho }\end{array}$ & $180 \pm 0$ & $180 \pm 0$ & $180 \pm 0$ & $178,9 \pm 2,4$ & $178,8 \pm 2,1$ \\
\hline & $\begin{array}{l}\text { Flexão } \\
\text { Plantar }\end{array}$ & $53,9 \pm 13,3$ & $53,2 \pm 12,2$ & $54,9 \pm 12,3$ & $54,2 \pm 14,5$ & $55,6 \pm 15,2$ \\
\hline & $\begin{array}{l}\text { Dorsi } \\
\text { Flexão }\end{array}$ & $14,2 \pm 6,3$ & $14,4 \pm 6,3$ & $16,6 \pm 7,4$ & $15,4 \pm 7,3$ & $15,5 \pm 7,3$ \\
\hline
\end{tabular}

TABELA 2. MÉDIA E DESVIO-PADRÃO DOS VALORES DE PERIMETRIA DA PERNA AVALIADA.

\begin{tabular}{ccccccc}
\hline & & Pré & Pós & 24 horas & 48 horas & 72 horas \\
\hline & Ponto 1 & $33,3 \pm 1,97$ & $33,4 \pm 1,92$ & $33,3 \pm 2,01$ & $33,3 \pm 2,05$ & $33,2 \pm 2,04$ \\
& Ponto 2 & $33,9 \pm 1,87$ & $34,5 \pm 2,03$ & $34,1 \pm 1,91$ & $34,3 \pm 2,07$ & $34,2 \pm 2,1$ \\
$\begin{array}{c}\text { Grupo } \\
\text { Controle }\end{array}$ & Ponto 3 & $32,6 \pm 1,89$ & $33,2 \pm 2,10$ & $32,5 \pm 2,02$ & $32,8 \pm 2,08$ & $32,6 \pm 2,19$ \\
& Ponto 4 & $28,9 \pm 1,99$ & $29,0 \pm 1,94$ & $28,6 \pm 1,96$ & $28,9 \pm 1,99$ & $28,8 \pm 1,95$ \\
& Ponto 5 & $24,9 \pm 1,69$ & $25,0 \pm 1,83$ & $24,5 \pm 1,73$ & $24,9 \pm 1,85$ & $24,9 \pm 1,95$ \\
& Ponto 6 & $21,8 \pm 1,23$ & $21,9 \pm 1,29$ & $21,7 \pm 1,27$ & $21,8 \pm 1,45$ & $21,8 \pm 1,35$ \\
\hline & Ponto 1 & $32,9 \pm 2,67$ & $33,0 \pm 2,21$ & $32,4 \pm 2,15$ & $32,4 \pm 2,06$ & $32,4 \pm 2,22$ \\
& Ponto 2 & $33,8 \pm 2,18$ & $34,4 \pm 2,21$ & $33,9 \pm 2,01$ & $34,0 \pm 2,1$ & $33,9 \pm 2,24$ \\
Grupo & Ponto 3 & $32,6 \pm 2,31$ & $33,1 \pm 2,28$ & $32,8 \pm 2,25$ & $32,9 \pm 2,1$ & $32,8 \pm 2,43$ \\
Massoterapia & Ponto 4 & $28,8 \pm 2,41$ & $29,1 \pm 2,32$ & $29,0 \pm 2,30$ & $28,9 \pm 2,2$ & $29,0 \pm 2,8$ \\
& Ponto 5 & $24,8 \pm 2,11$ & $24,9 \pm 2,08$ & $24,8 \pm 2,05$ & $24,8 \pm 1,83$ & $24,9 \pm 2,1$ \\
& Ponto 6 & $21,6 \pm 1,56$ & $21,6 \pm 1,56$ & $21,5 \pm 1,45$ & $21,6 \pm 1,42$ & $21,6 \pm 1,48$ \\
\hline
\end{tabular}




\section{DISCUSSÃO}

O principal objetivo do presente estudo foi investigar o efeito da massoterapia nos sintomas da dor muscular tardia. Para isso, foram aplicados 30 minutos de massagem realizada imediatamente após o exercício excêntrico, sendo avaliada a amplitude de movimento, a presença do edema e a intensidade da dor. Os resultados sugerem que o protocolo foi eficaz na prevenção da redução da amplitude do movimento de extensão ativa do joelho, entretanto, o mesmo não foi eficaz na diminuição da dor.

$O$ protocolo de massagem realizado neste estudo parece ter sido eficaz na redução da perda de amplitude de movimento, visto que a pressão mecânica aplicada sobre o músculo tríceps sural diminuiu a rigidez músculo-tendínea ${ }^{(23)}$ permitindo a manutenção da flexibilidade tecidual. Entretanto, alguns estudos questionam a eficácia da massagem na manutenção da ADM após a indução da dor muscular tardia, isso se deve as limitações metodológicas e técnicas utilizadas ${ }^{(7,8)}$. Neste estudo fazendo o uso do deslizamento superficial, petrissage e effleurage durante 30 minutos logo após o exercício parece ter evitado a perda de amplitude de movimento, contrastando com o trabalho de Hilbert et al $^{(7)}$ que aplicou 2 horas após o exercício 20 minutos de effleurage, tapotagem e petrissage; e o de Zainuddin et al $^{(8)}$ que aplicou 3 horas após o exercício 10 minutos das técnicas de effleurage, fricção e petrissage e não obtiveram diminuição da perda de amplitude de movimento. Os resultados obtidos devem estar relacionados às técnicas utilizadas, já que o presente estudo não fez uso da técnica de tapotagem, devido à mesma estimular uma ação reflexa do fuso muscular ${ }^{(24)}$, o que poderia aumentar a rigidez muscular; e nem da fricção que tem como objetivo quebrar adesões de lesões antigas $^{(25)}$, o qual não se encaixa no tratamento da dor muscular tardia que é causada por microlesões recentes. As demais técnicas utilizadas têm como principais objetivos o relaxamento e mobilização do tecido muscular e subcutâneo ${ }^{(26)}$.

No entanto, os resultados encontrados na literatura e no presente estudo podem ter sido influenciados pela variabilidade inerente da medida goniométrica manual. Sugerindo assim, a realização de estudos que utilizem instrumentos mais precisos, afim de elucidar realmente o efeito de tais recursos na minimização da redução de ADM em casos de dor muscular tardia.

Segundo nossos resultados, o grupo controle apresentou evolução da dor como encontrado na literatura, com picos de 48 horas $^{(10,11,12)}$, mostrando que o protocolo de exercício para indução de dor foi positivo neste estudo. A massagem utilizada logo após o exercício excêntrico não obteve resultados favoráveis quanto à redução da dor, isto possivelmente se deve ao fato de que a massagem aplicada imediatamente agiu como um fator pró-inflamatório, devido a uma vasodilatação inicial pela estimulação do sistema nervoso parassimpático ${ }^{(26)}$, conseqüentemente um aumento da circulação sanguínea(1), bem como da permeabilidade destes vasos facilitando a migração de células inflamatórias ao local das microlesões que ao liberarem prostaglandinas aumentaram a sensibilidade dos receptores de dor $^{(4)}$. Já ao iniciar a massagem 2 horas após o exercício, mesmo com duração de aplicação diferentes, estudos comprovaram a eficácia da massagem na redução da dor, tendo Smith et al (22) aplicado 30 minutos de massagem, enquanto Rodenburg et $\mathrm{al}^{(27)}$, aplicou 15 minutos de massagem obtendo resultados em comum, como a redução da dor muscular tardia e da creatina quinase, mostrando que de acordo com o tempo que a massagem é iniciada, ela age de maneira diferente no processo inflamatório, podendo contribuir ou reduzir com a inflamação.

Logo, a massagem pode ser efetiva na redução da dor muscular tardia, dependendo do tempo que se inicia a aplicação da técnica, bem como na prevenção da diminuição da amplitude de movimento, estando os efeitos relacionados às técnicas utilizadas; sendo, portanto, importante que novos estudos verifiquem a eficácia do protocolo de massagem utilizado neste artigo aplicado algumas horas após o exercício excêntrico.

\section{CONCLUSÃO}

No tratamento de dor muscular tardia, a massoterapia aplicada logo após o exercício excêntrico parece ser eficaz na redução da perda de amplitude de movimento de extensão ativa do joelho; porém não reduz a dor que 
tem o seu pico em 48 horas. Entretanto, é necessária a realização de novos estudos que avaliem diferentes grupos musculares, em outras populações e que principalmente utilizem métodos mais precisos que o goniômetro manual para finalmente determinar os verdadeiros efeitos da massoterapia na manutenção da ADM na presença da dor muscular tardia.

\section{AGRADECIMENTOS}

Nós agradecemos à direção da academia Espaço Fitness por disponibilizar os aparelhos e o espaço para a realização dessa pesquisa e aos voluntários que foram importantes instrumentos para o desenvolvimento da mesma.

\section{REFERÊNCIAS BIBLIOGRÁFICAS}

1. Armstrong RB. Mechanisms of exercise induced delayed onset muscular soreness: a brief review. Medicine and Science in Sports and Exercise 1984; 16 : 529-538.

2. Maclntyre DL, Reid WD, McKenzie DC. Delayed onset muscle soreness: the inflammatory response to muscle injury and its clinical implications. Sports Med 1995; 20:24-40.

3. Powers SK, Howley ET. Exercise physiology: theory and application to fitness and performance. 3rd ed. Baltimore, MD: Williams \& Waverly,1996.

4. Smith LL. Acute inflammation: the underlying mechanisms in delayed onset muscle soreness? Med Sci Sports Exerc 1991;23:542-51.

5. Herbert RD; DE Noronha M. Stretching to prevent or reduce muscle soreness after exercise (Review). 4. ed. The Cochrane Collaboration, 2007. 25p.

6. Athanasios Z, Theocharis V, Tofas T, Tsiokanos A, Yfanti C, Paschalis V, Koutedakis Y, Nosaka K. Comparison between leg and arm eccentric exercises of the same relative intensity on indices of muscle damage. Eur J Appl Physiol 2005 95: 179-185.

7. Hilbert JE, Sforzo GA, Swensen T. The effects of massage on delayed onset muscle soreness. $\mathrm{Br} J$ Sports Med 2003;37:72-75.

8. Zainuddin Z, Newton N, Sacco P, Nosaka K. Effects of massage on delayed-onset muscle soreness, swelling, and recovery of muscle function. J Ath Train 2005;40(3):174-180
9. Howell JN, Chila AGA, Ford $G$, et al. An electromyographic study of elbow motion during postexercise muscle soreness. J Appl Phyosiol 1985; 58 (5): 1711-8.

10. Talag T. Residual muscle soreness as influenced by concentric, eccentric, and static contractions. Res $Q$ 1973; 44:458-69

11. Jones DA; Newham DJ; Round JM. Experimental human muscle damage: morphological changes in relation to other indices of damage. $J$ Physiol 1986;375:435-48.

12. Cleak M; Eston RG. Delayed onset muscle soreness: mechanisms and management. J Sports Sci 1992; 10 (4):325-41.

13. Armstrong RB, Warren GL, Warren JA. Mechanisms of exercise-induced muscle fibre injury. Sports Med. 1991; 12: 184-207.

14. Knight KL. Crioterapia no Tratamento das Lesões Desportivas. $1^{a}$ ed. São Paulo: Manole; 2000.

15. Mayer JM, Mooney V, Matheson LN, Erasala GN, Verna JL, Udermann BE, Leggett S. Continuous lowlevel heat wrap therapy for the prevention and early phase treatment of delayed onset muscle soreness of the low back : a randomized controlled trial. Arch Phys Med Rehabil 2006; 87(10): 1310-1317

16. Sellwood KL, Brukner $P$, Williams D, Nicol A, Hinman R. Ice-water immersion and delayed-onset muscle soreness: a randomised controlled trial. $\mathrm{Br} \mathrm{J}$ Sports Med 2007; 41: 392-397.

17.Gulick DT, Kimura IF. Delayed onset muscle soreness: what is it and how do we treat it? J Sport Rehab 1996; 5: 234-243.

18. Stay J C, Ricard M D, Draper D O, Schulthies S S, Durrant E. Pulsed ultrasound fails to diminish delayedonset muscle soreness symptoms. Journal of Athletic Training 1998; 33(4): 341-6.

19. Ernst E. Does post-exercise massage treatment reduce delayed onset muscle soreness? A systematic review. $\mathrm{Br}$ J Sports Med 1998;32:212-214.

20. Farr, T., Nottle, C., Nosaka, K., \& Sacco, P. The effects of therapeutic massage on delayed onset muscle soreness and muscle function following downhill walking. Journal Of Science and Medicina in Sport 2002; 5:297-306.

21. Hilbert JE, Sforzo GA, Swensen T: The effects of massage on delayed onset muscle soreness. $\mathrm{Br} \mathrm{J}$ Sports Med 37:72-75, 2003 
22. Smith LL,Keating MN, Holbert D, et al. The effects of athletic massage on delayed onset muscle soreness, creatina Kinase and neutrophil count: a preliminary report. Journal of Orthopedic and Sport Physical Therapy 1994:19 (2):93-9.

23. Rodenburg, J., Steenbeek, D., Schiereck, P., \& Bar, P. Warm-up, stretching and massage diminish harmful effects of eccentric exercise. International Journal of Sports Medicine 1994; 15:414-419.

24. Armstrong R. Initial events in exercise-induced muscular injury. Med Sci Sports Exerc 1990; 22(4): 429435.

25. Miles MP, Clarkson PM. Exercise-induced muscle pain, soreness, and cramps. J Sports Med Phys Fit. 1994;34: 203-216.
26. Schwane JA, Hatrous BG, Johnson SR, et al. Is lactic acid related to delayed-onset muscle soreness? Phys Sports Med 1983; 11(3): 124-127, 130-131.

27. De Domenico G. Técnicas de Massagem de Beard. 4. ed. São Paulo: Manole, 1998.

Endereço para correspondência:

Prof. Rodrigo Cappato de Araújo

Universidade de Pernambuco - Campus Petrolina BR 203 Km2 S/N

Vila Eduardo - Petrolina - PE

CEP 56.300-000

Email: rodrigo.cappato@upe.br labracjuliane@hotmail.com Ipls2002@hotmail.com charlesvieira.petrolina@yahoo.com.br 